\title{
Article \\ Synthesis and Characterization of Nanomaterial Based on Halloysite and Hectorite Clay Minerals Covalently Bridged
}

\author{
Marina Massaro ${ }^{1}\left(\mathbb{D}\right.$, Cesar Viseras Iborra ${ }^{2,3, *}$, Giuseppe Cavallaro ${ }^{4,5}\left({ }^{\circledR}\right.$, Carmelo Giuseppe Colletti ${ }^{1}$, \\ Fátima García-Villén ${ }^{2}\left(0\right.$, Giuseppe Lazzara ${ }^{4,5}$ and Serena Riela ${ }^{1, *}$
}

1 Department of Biological, Chemical and Pharmaceutical Sciences and Technologies, University of Palermo Viale delle Scienze, Ed. 17, 90128 Palermo, Italy; marina.massaro@unipa.it (M.M.); carmelogiuseppe.colletti@unipa.it (C.G.C.)

2 Department of Pharmacy and Pharmaceutical Technology, Faculty of Pharmacy, University of Granada, Campus of Cartuja, 18071 s/n Granada, Spain; fgarvillen@ugr.es

3 Andalusian Institute of Earth Sciences, CSIC-UGR. Avenida de las Palmeras 4, 18100 Granada, Spain

4 Department of Physics and Chemistry, University of Palermo, 90128 Palermo, Italy; giuseppe.cavallaro@unipa.it (G.C.); giuseppe.lazzara@unipa.it (G.L.)

5 National Interuniversity Consortium of Materials Science and Technology (INSTM), Research Unit of Palermo, Via G. Giusti, 9, 50121 Florence, Italy

* Correspondence: cviseras@ugr.es (C.V.I.); serena.riela@unipa.it (S.R.)

check for updates

Citation: Massaro, M.; Viseras Iborra, C.; Cavallaro, G.; Colletti, C.G.; García-Villén, F.; Lazzara, G.; Riela, S. Synthesis and Characterization of Nanomaterial Based on Halloysite and Hectorite Clay Minerals Covalently Bridged. Nanomaterials 2021, 11, 506. https://doi.org/ 10.3390/nano11020506

Academic Editor: Montserrat Gómez

Received: 26 January 2021

Accepted: 15 February 2021

Published: 17 February 2021

Publisher's Note: MDPI stays neutral with regard to jurisdictional claims in published maps and institutional affiliations.

Copyright: (c) 2021 by the authors. Licensee MDPI, Basel, Switzerland. This article is an open access article distributed under the terms and conditions of the Creative Commons Attribution (CC BY) license (https:// creativecommons.org/licenses/by/ $4.0 /)$.

\begin{abstract}
Halloysite is an aluminosilicate clay with a predominantly hollow tubular structure (HNTs) able to act as a nanocontainer for the encapsulation of several chemicals. However, HNTs possess low affinity for metal ions in their pristine form and they need to be modified for improving their adsorption capabilities. Therefore, to overcome this issue herein we report a straightforward approach for the covalent modification of the external surface of halloysite nanotubes with hectorite clay. Compared to halloysite, hectorite possesses a lamellar structure with higher cation exchange capacity. The covalent linkage between the two clays was verified by several techniques (FTIR spectroscopy, ${ }^{13}$ C CP-MAS NMR, TGA, $\zeta$-potential, DLS, and XRD measurements) and the morphology was imaged by TEM investigations. As proof of concept the adsorption ability of the obtained nanomaterial in comparison to pristine clays was proved using ciprofloxacin and silver ions chosen as models for their different chemical characteristics.
\end{abstract}

Keywords: halloysite nanotubes; hectorite; covalent linkage; synthetic strategy

\section{Introduction}

Clay minerals are natural phyllosilicates which present peculiar morphologies that are a challenge for chemical manipulation. Furthermore, their combination at the microor nanometric scale plays an important role as scaffold for the development of appealing artificial nanocomposites for several applications ranging from industry to biomedical engineering. Halloysite is an aluminosilicate with a predominantly hollow tubular structure (HNTs) [1]. It is dioctahedral 1:1 clay mineral present in the soil, belonging to the kaolin group. Halloysite possesses different charged surfaces: positive in the inner lumen, where mostly of aluminum hydroxide groups are present; negative on the external surface, which consists in silicon dioxide. HNTs can be selectively functionalized at the inner and/or outer surfaces leading to the synthesis of several interesting nanomaterials [2-6]. The most important feature of HNTs is their empty lumen which acts as a nanocontainer for the encapsulation of several biologically active species $[4,7,8]$. However, HNTs possess low affinity for metal ions in their pristine form and they need to be modified for improving their adsorption capabilities $[9,10]$. In the years, several approaches have been exploited but few of them envisage the possibility to delivery simultaneously two or more active ingredients. 
Hectorite $(\mathrm{Ht})$, a natural clay mineral belonging to the smectite group with a general formula of $\mathrm{Na}_{0.3}\left(\mathrm{Mg}, \mathrm{Li}_{3} \mathrm{Si}_{4} \mathrm{O}_{10}(\mathrm{OH})_{2}\right.$ possesses a lamellar structure and due to this it shows higher cation exchange capacity [11].

Hence, the combination of HNTs and Ht could improve halloysite properties by the development of functional nanoarchitectures which could benefit, in terms of adsorption abilities, thermal stability, solvent resistance and so on, of both HNTs and Ht properties.

Glotov and Lvov, for example, reported a self-assembly strategy via centhyltrimethyl ammonium bromide onto negative HNTs surface, to obtain MCM-41/HNTs material with enhanced thermal and mechanical stability [12]. An example of chemical mixture between two clay was reported by $\mathrm{Mu}$ and Wang [13]. In this case palygorskite and halloysite were combined to improve betain adsortpion. They highlighted that the introduction of halloysite in a palygorskite mixture enhances the physico-chemical properties of palygorskite, such as color performance, heating stability, and solvent resistance. To the best of our knowledge a limited number of publications deals with the preparation of functional materials through the cross-assembly of different components but no one reports the covalent linkage between two clays [14]. This limitation is probably due to the repulsive interactions existing between the negative charges present on the clays external surface. Conversely to the physical combination of clays, a covalent linker among them, could confer to the final nanomaterial new properties, as robustness, which could improve, for example the manufacturing process of pharmaceutical products, by reducing operating cost and improving final product quality.

Herein, we report a bottom-up approach to synthetize a multifunctional nanomaterial based on halloysite covalently bridged to hectorite (HNTs-Ht) (Scheme 1), that could highlight the peculiarity of the single clays simultaneously. Indeed, by considering the above-mentioned properties of the two clay minerals, the incorporation of Ht in HNTs external surface is made with a specific goal: to bring or complement missing properties of HNTs, as adsorption ability towards metal ions. The covalent linkage between the two clays was verified by several techniques (FTIR spectroscopy, ${ }^{13} \mathrm{C}$ CP-MAS NMR, TGA, $\zeta$-potential, DLS and XRD measurements) and the morphology was imaged by TEM investigations. As proof of concept the adsorption ability of HNTs-Ht compared to pristine clays was proved using two antibacterial agents such as ciprofloxacin and silver ions as models.

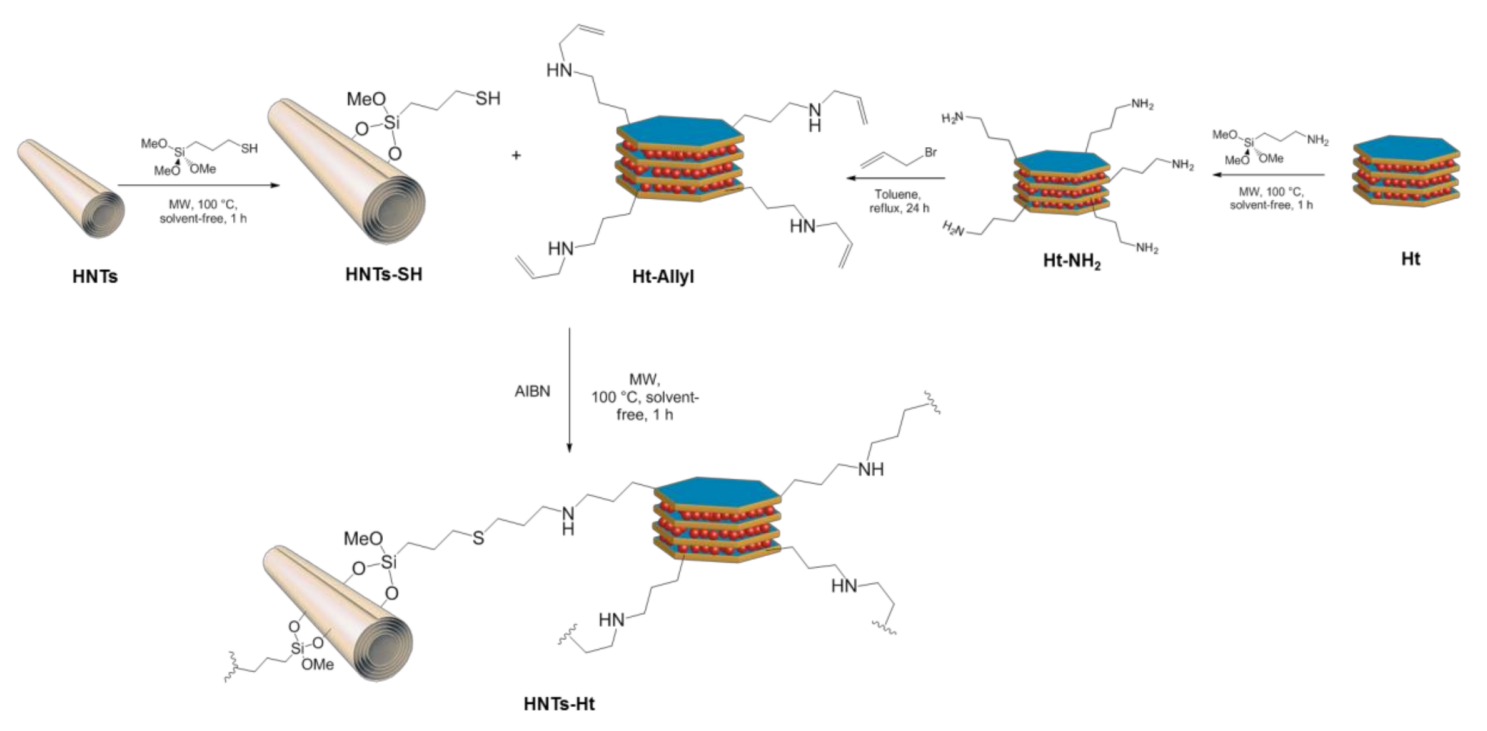

Scheme 1. Schematic representation of HNTs-Ht nanomaterial synthesis. 


\section{Materials and Methods}

All reagents needed were purchased from Sigma-Aldrich and used without further purification.

Hectorite was kindly gifted by Tolsa Group Inc. (Madrid, Spain).

Thermogravimetric analyses were performed on a Q5000 IR apparatus (TA Instruments, New Castle, DE, USA) under a nitrogen flow of $25 \mathrm{~cm}^{3} \mathrm{~min}^{-1}$ for the sample and $10 \mathrm{~cm}^{3} \mathrm{~min}^{-1}$ for the balance. The weight of each sample was ca. $10 \mathrm{mg}$. Measurements were carried out by heating the sample from room temperature up to $900{ }^{\circ} \mathrm{C}$ at a rate of $10{ }^{\circ} \mathrm{C} \min ^{-1}$.

FTIR spectra (KBr) were recorded with an Agilent Technologies Cary 630 FTIR spectrometer (Agilent Technologies, Santa Clara, CA, USA). Specimens for these measurements were prepared by mixing $5 \mathrm{mg}$ of the sample powder with $100 \mathrm{mg}$ of $\mathrm{KBr}$. FTIR spectrum of the HNTs-Ht nanomaterial was recorded on a JASCO6200 spectrophotometer (software Spectra Manager II). All samples were studied in the $400-4000 \mathrm{~cm}^{-1}$ range, with a resolution of $2 \mathrm{~cm}^{-1}$.

Microwave-assisted syntheses were carried out with a CEM DISCOVER monomode system (CEM Corporation, Matthews, NC, USA) in a closed vessel.

${ }^{13}$ C CP-MAS NMR spectra were acquired on a Bruker Advance II 400 spectrometer (Bruker, Billerica, MA, USA) operating at $100.63 \mathrm{MHz}$ for ${ }^{13} \mathrm{C}$ nuclides and $400.15 \mathrm{MHz}$ for $1 \mathrm{H}$ nuclides equipped with a $4 \mathrm{~mm}(\mathrm{H}-\mathrm{X})$ double channel CPMAS probe. The samples were placed in a $4 \mathrm{~mm}$ zirconia rotor closed with Kel-F caps. The spectra were measured at a MAS speed of $8 \mathrm{kHz}$, with $2 \mathrm{~ms}$ contact time, $3 \mathrm{~s}$ delay time and an excitation pulse of $4.7 \mathrm{~ms}$ on the $1 \mathrm{H}$ nucleus, and 1024 scans were performed. The Hartmann-Hahn-condition was optimized using an adamantane standard sample, which was also used as an external chemical shift reference.

Transmission electron microscopy (TEM) was performed by means of a FEI Titan G2 60-300 ultra-high resolution transmission electron microscope (FEI, Lausanne, Switzerland) coupled with analytical electron microscopy (AEM) performed with a SUPER-X silicondrift windowless energy-dispersive X-ray spectroscopy (XEDS) detector. AEM spectra were saved in mode STEM (scanning transmission electron microscopy) with a HAADF (high angle annular dark field) detector. X-ray chemical element maps were also collected.

X-ray powder diffraction (XRPD) analysis was carried out using a diffractometer (X'Pert Pro model, Malven Panalytical, Madrid, Spain) equipped with a solid-state detector ( $\mathrm{X}^{\prime}$ Celerator) and a spinning sample holder. The diffractogram patterns were recorded using random oriented mounts with $\mathrm{CuK} \alpha$ radiation, operating at $45 \mathrm{kV}$ and $40 \mathrm{~mA}$, in the range $4-60^{\circ} 2 \theta$. HNTs-SH material was synthetized as reported elsewhere [15].

\subsection{Synthesis of $\mathrm{Ht}-\mathrm{NH}_{2}$}

A total of $0.500 \mathrm{~g}$ of Ht were weighed in an MW test tube provided with a cap, and $1 \mathrm{~mL}$ of 3-aminopropyltrimethoxysilane was added dropwise. The mixture was dispersed ultrasonically for $30 \mathrm{~min}$ at room temperature and inserted in an MW apparatus at $100{ }^{\circ} \mathrm{C}$ under constant stirring for $1 \mathrm{~h}$. The powder was filtered, rinsed with $\mathrm{MeOH}$ and dried at $80^{\circ} \mathrm{C}$ under vacuum.

\subsection{Synthesis of Ht-Allyl}

In a round bottom flask $0.100 \mathrm{~g}$ of $\mathrm{Ht}-\mathrm{NH}_{2}$ (which correspond to $0.10 \mathrm{mmol}$ of $-\mathrm{NH}_{2}$ groups) were dispersed in $10 \mathrm{~mL}$ of dry toluene and $100 \mu \mathrm{L}(0.20 \mathrm{mmol}, 2 \mathrm{eq})$ of allyl bromide were added. The mixture was sonicated for $5 \mathrm{~min}$, and then stirred at $110{ }^{\circ} \mathrm{C}$ for $24 \mathrm{~h}$. Afterwards, the crude solid was filtered, washed with several aliquots of $\mathrm{MeOH}$ and dried overnight at $80^{\circ} \mathrm{C}$ under vacuum. 


\subsection{Synthesis of HNTs-Ht}

HNTs-SH (50 mg) and Ht-Allyl $(50 \mathrm{mg})$ were weighed in a MW test tube provided with a cap and a catalytic amount of AIBN was added. The mixture was inserted in the MW apparatus at $100{ }^{\circ} \mathrm{C}$, under constant stirring, for $1 \mathrm{~h}$. Successively, the solid was filtered off, rinsed several times with $\mathrm{CH}_{2} \mathrm{Cl}_{2}$ and $\mathrm{MeOH}$ and dried at $80^{\circ} \mathrm{C}$ under vacuum.

\subsection{Loading of Ciprofloxacin on HNTs, Ht and HNTs-Ht}

To a dispersion of HNTs, Ht or HNTs-Ht (see infra) in methanol $(5 \mathrm{~mL})$, a ciprofloxacin solution $\left(1 \mathrm{~mL}, 1 \times 10^{-2} \mathrm{M}\right.$ in $\mathrm{HCl} 0.1 \mathrm{~N}$ ) was added. The suspension was sonicated for $5 \mathrm{~min}$, at an ultrasound power of $200 \mathrm{~W}$ and at $25^{\circ} \mathrm{C}$ and was left under stirring for $18 \mathrm{~h}$ at room temperature. After this time, the solvent was filtered off, under vacuum, and the powder was washed several times with methanol and then dried.

\subsection{Loading of Silver Ions on HNTs, Ht and HNTs-Ht}

To a $\mathrm{AgNO}_{3}$ aqueous solution ( $\left.5 \mathrm{~mL}, 1 \times 10^{-2} \mathrm{M}\right)$, HNTs, Ht or HNTs-Ht (see infra) were added and the obtained dispersions were left to stir at room temperature for ca. $18 \mathrm{~h}$. After this time, the solvent was filtered off and the powder was washed with water $(3 \times 5 \mathrm{~mL})$. The powders were dried at $60^{\circ} \mathrm{C}$ overnight and the supernatant solutions were analyzed by $\mathrm{AgCl}$ test to determine the $\mathrm{Ag}^{+}$ions amount loaded on the clays. In detail, the supernatant solutions were titrated with a standard solution of $\mathrm{NaCl}(0.01 \mathrm{M})$ in the presence of dichlorofluorescein, used as indicator. After the endpoint was reached the concentration of $\mathrm{Ag}^{+}$was determined by the following equation:

$$
\mathrm{C}_{1} \mathrm{~V}_{1}=\mathrm{C}_{2} \mathrm{~V}_{2}
$$

where $\mathrm{C}_{1}$ and $\mathrm{V}_{1}$ are the concentration of the $\mathrm{NaCl}$ solution and the added volume, respectively, while $C_{2}$ and $V_{2}$ are the concentration and volume of $\mathrm{Ag}^{+}$ions in the supernatant solution.

\section{Results and Discussion}

The HNTs-Ht nanomaterial was obtained by reaction between complementary groups grafted to HNTs external surface and modified Ht edges, thiol and allyl, respectively. By means of this synthetic pathway we obtain the best conditions to minimize repulsive interactions that occur between halloysite and hectorite external surfaces (both negatively charged).

\subsection{Synthesis and Characterization of Ht-Allyl Nanomaterial}

Functionalized HNTs by thiol groups was obtained by a procedure reported in literature [15], while Ht was functionalized with allyl units in a two steps procedure (Scheme 1). First, the silanol groups present at the edges of the Ht clay were reacted with an excess of 3-amino propyltrimethoxysilane to obtain an amino functionalized $\mathrm{Ht}\left(\mathrm{Ht}-\mathrm{NH}_{2}\right)$. This reaction was carried out following experimental procedure previously adopted by us for similar systems [16]. In detail, the grafting of the organosilane at the clay edges was performed by microwave (MW) irradiation at $100{ }^{\circ} \mathrm{C}$, in solvent free conditions, for an irradiation time of $1 \mathrm{~h}$. After work-up, the degree of functionalization of the clay edges, estimated by thermogravimetric analysis (TGA), was $1.03 \mathrm{mmol} \mathrm{g}^{-1}$.

The Ht- $\mathrm{NH}_{2}$ material was further modified by means of a nucleophilic substitution among the amino groups at $\mathrm{Ht}$ edges and allyl bromide, in toluene, at $110{ }^{\circ} \mathrm{C}$ for a reaction time of $24 \mathrm{~h}$, to give the compound Ht-Allyl with a degree of functionalization of $2.08 \mathrm{mmol} \mathrm{g}^{-1}$ (Scheme 1). The successful functionalization was confirmed by FTIR spectroscopy and TGA.

In Figure $1 \mathrm{a}$ are reported the FTIR spectra of $\mathrm{Ht}-\mathrm{NH}_{2}$ and $\mathrm{Ht}-\mathrm{Allyl}$ compounds and for comparison that for pristine Ht. The FTIR spectrum of pristine Ht showed all characteristic bands of the clay at ca. $3750-3150 \mathrm{~cm}^{-1}$, assigned to the stretching vibration of structural O-H (Mg-O-H and Si-O-H) and adsorbed water. The band at $1635 \mathrm{~cm}^{-1}$ is attributed to bending modes of $-\mathrm{OH}$ in adsorbed water molecule directly coordinated to 
the exchangeable cations of the clay mineral and the one at $1020 \mathrm{~cm}^{-1}$ is associated to the Si-O-Si stretching vibrations [17]. Compared to pristine $\mathrm{Ht}, \mathrm{Ht}-\mathrm{NH}_{2}$ exhibits, in the FTIR spectrum, the vibration band for C-H stretching of methylene groups around $2980 \mathrm{~cm}^{-1}$ and a band around $3200 \mathrm{~cm}^{-1}$ due to the vibration stretching bands of the primary $-\mathrm{NH}_{2}$ groups of the grafted aminosilane and the vibration band at $\sim 1490 \mathrm{~cm}^{-1}$ due to the bending vibrations of the $-\mathrm{NH}$ - groups. After reaction with allyl bromide new signals appeared in the FTIR spectrum of the final nanomaterial. In particular is clearly evident the band at $\sim 1560 \mathrm{~cm}^{-1}$ that corresponds to the vibration stretching band of $\mathrm{C}=\mathrm{C}$ bonds and the signal at $\sim 1350 \mathrm{~cm}^{-1}$ due to the vinylidene $\mathrm{C}-\mathrm{H}$ in-plane bending.

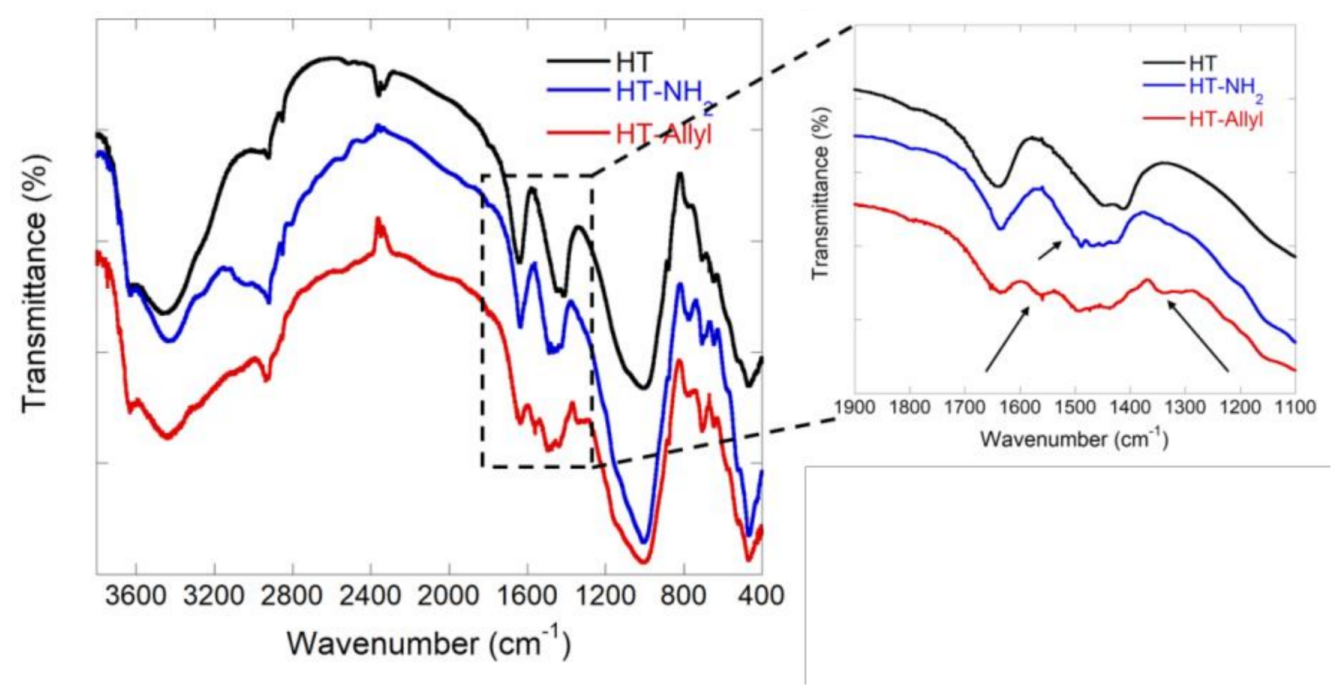

(a)

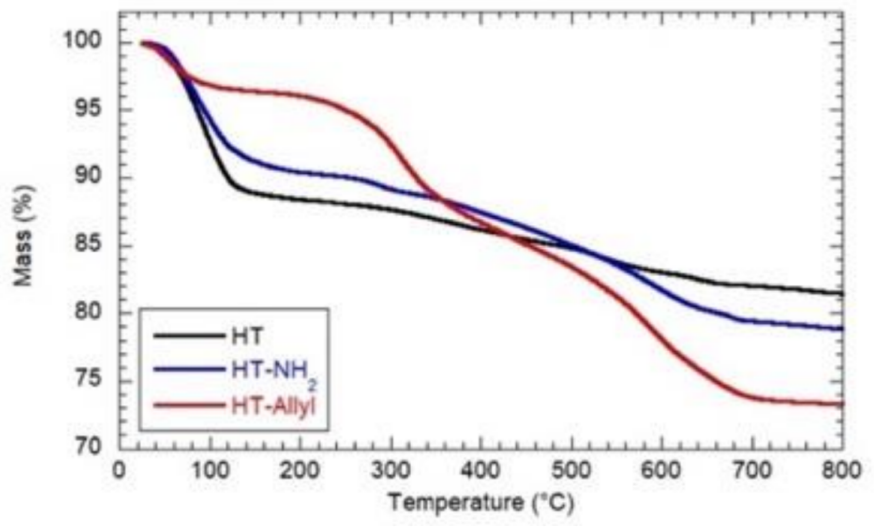

(b)

Figure 1. (a) FTIR spectra of pristine $\mathrm{Ht}, \mathrm{Ht}_{-} \mathrm{NH}_{2}$ and $\mathrm{Ht}-\mathrm{Allyl}$; and TG curves (b) for pristine $\mathrm{Ht}, \mathrm{Ht}-\mathrm{NH}_{2}$ and $\mathrm{Ht}-\mathrm{Allyl}$.

In Figure $1 \mathrm{~b}$ are reported the thermogravimetric curves for $\mathrm{Ht}, \mathrm{Ht}-\mathrm{NH}_{2}$ and $\mathrm{Ht}-\mathrm{Allyl}$ materials. The analysis of TG curves allowed us to calculate the mass loss from room temperature to $150{ }^{\circ} \mathrm{C}\left(\mathrm{ML}_{150}\right)$ and the residual mass at $700{ }^{\circ} \mathrm{C}\left(\mathrm{MR}_{800}\right)$. Based on these results, we calculated the degraded mass at $700{ }^{\circ} \mathrm{C}\left(\mathrm{MD}_{800}\right)$ as $100-\left(\mathrm{ML}_{150}+\mathrm{MR}_{800}\right)$. The thermogravimetric parameters are summarized in Table 1. 
Table 1. Thermogravimetric parameters for HT, HT- $\mathrm{NH}_{2}$, and HT-Allyl.

\begin{tabular}{cccc}
\hline Material & $\mathbf{M L}_{\mathbf{1 5 0}}(\mathbf{w t} \%)$ & $\mathbf{M R}_{\mathbf{8 0 0}}(\mathbf{w t} \%)$ & $\mathbf{M D}_{\mathbf{8 0 0}} \mathbf{( w \mathbf { t } \% )}$ \\
\hline HT & 11.09 & 82.0 & 6.91 \\
HT-NH & 8.76 & 79.4 & 11.84 \\
HT-Allyl & 3.58 & 73.8 & 22.62 \\
\hline
\end{tabular}

According to literature [18], $\mathrm{ML}_{150}$ reflects the moisture content of the material. Consequently, this parameter is related to its hydrophilicity. As highlighted in Table 1, the covalent modification of $\mathrm{Ht}$ surfaces reduces the hydrophilicity of the nanoclay. In addition, the increase of $\mathrm{MD}_{800}$ value in the $\mathrm{Ht}-\mathrm{NH}_{2}$ and Ht-Allyl materials can be attributed to the presence of organic components proving the successful functionalization of hectorite.

\subsection{Synthesis and Characterization of HNTs-Ht Nanomaterial}

The covalently linked HNTs-Ht nanomaterial was prepared by reacting the Ht-Allyl nanomaterial with HNTs-SH by AIBN-catalyzed thiol-ene reaction [19] in a 1:1 weight ratio (Scheme 1). The reaction was carried out by MW irradiation, in solvent free conditions, at $100{ }^{\circ} \mathrm{C}$ for an irradiation time of $1 \mathrm{~h}$. On the basis of the stoichiometric ratio, allyl groups on $\mathrm{Ht}$ were in excess relative to the moles of -SH groups onto HNTs (degree of functionalization of 0.35 and $1.03 \mathrm{mmol} \mathrm{g}^{-1}$ for HNTs-SH and Ht-Allyl, respectively), therefore the double bonds that do not react in the thiol-ene reaction can undergo a selfaddition reaction, leading to the formation of a coating onto HNTs surface, constituted by a Ht cross-linked network [20].

The HNTs-Ht nanomaterial was characterized by FTIR and ${ }^{13} \mathrm{C}$ CP-MAS NMR spectroscopies and TGA. In the FTIR spectrum of HNTs-Ht (Figure 2a) are shown all typical vibration bands of the clays precursors and in addition it showed the disappearance of the bands at ca. $1560 \mathrm{~cm}^{-1}$ and $1350 \mathrm{~cm}^{-1}$ indicating that the thiol-ene among thiol and allyl groups and the self-addition among different allyl groups reactions occurred.

In Figure $2 b$, the ${ }^{13} \mathrm{C}$ cross-polarization magic angle spinning $\left({ }^{13} \mathrm{C} C \mathrm{CP}-\mathrm{MAS}\right) \mathrm{NMR}$ spectra of the Ht-Allyl and HNTs-Ht nanomaterials are reported. In the solid-state NMR spectrum of Ht-Allyl nanomaterial (blue spectrum), the characteristic signals of the carbons of the allyl groups $\left(-\mathrm{CH}=\mathrm{CH}_{2}\right)$ between $150-110 \mathrm{ppm}$ are present, as well as those relative to the carbons of the methylene groups of the organic functionalities in the range 50-20 ppm. In addition, it is also present a signal at ca. $10 \mathrm{ppm}$ attributable to the $-\mathrm{CH}_{2}-\mathrm{Si}$ groups. After the covalent linkage, the solid-state NMR spectrum of HNTs-Ht (black spectrum) shows the disappearance of the signals relative to the allyl groups further confirming the successful covalent linkage between the two clays.

The thermogravimetric curve of HNTs-Ht (Figure S1) evidenced three clear degradation steps in the temperature ranges $25-150,200-400$ and $450-600{ }^{\circ} \mathrm{C}$. As previously reported, the mass loss at $25-150{ }^{\circ} \mathrm{C}(2.64 \mathrm{wt} \%)$ can be attributed to the moisture content. On the other hand, the mass decrease in the range between 200 and $400{ }^{\circ} \mathrm{C}(6.83 \mathrm{wt} \%)$ is related to the organic part of the Hal-Ht hybrid nanomaterial, while the signal change at $450-600{ }^{\circ} \mathrm{C}(7.95 \mathrm{wt} \%)$ is mostly due to expulsion of the interlayer water molecules of halloysite clay nanotubes [3].

DLS and $\zeta$-potential experiments were performed to investigate the effects of the synthesis procedure on the aqueous dynamic behavior and the surface charge of halloysite and hectorite clays.

We observed that pristine hectorite and halloysite possess similar hydrodynamic diameter values (Table 2). The surface modification with allyl and SH groups (for Ht and HNTs, respectively) slightly affects the aqueous dynamic behavior of both nanoclays. In addition, the hydrodynamic diameter of HNTs-Ht is comparable with those of the linked clays. For a correct evaluation of the hydrodynamic diameter, one should keep in mind that these quantities are related to the translational diffusion of the equivalent sphere and therefore are dominated by the larger characteristic size in anisotropic particles. In other words, clustering side-by-side some nanotubes would not affect much the observed 
values [21]. The constant value of the hydrodynamic size for the linked systems indicates that the network is not extended toward all mass of both materials, instead, small clusters containing a limited number of HNTs/Ht particles are obtained.

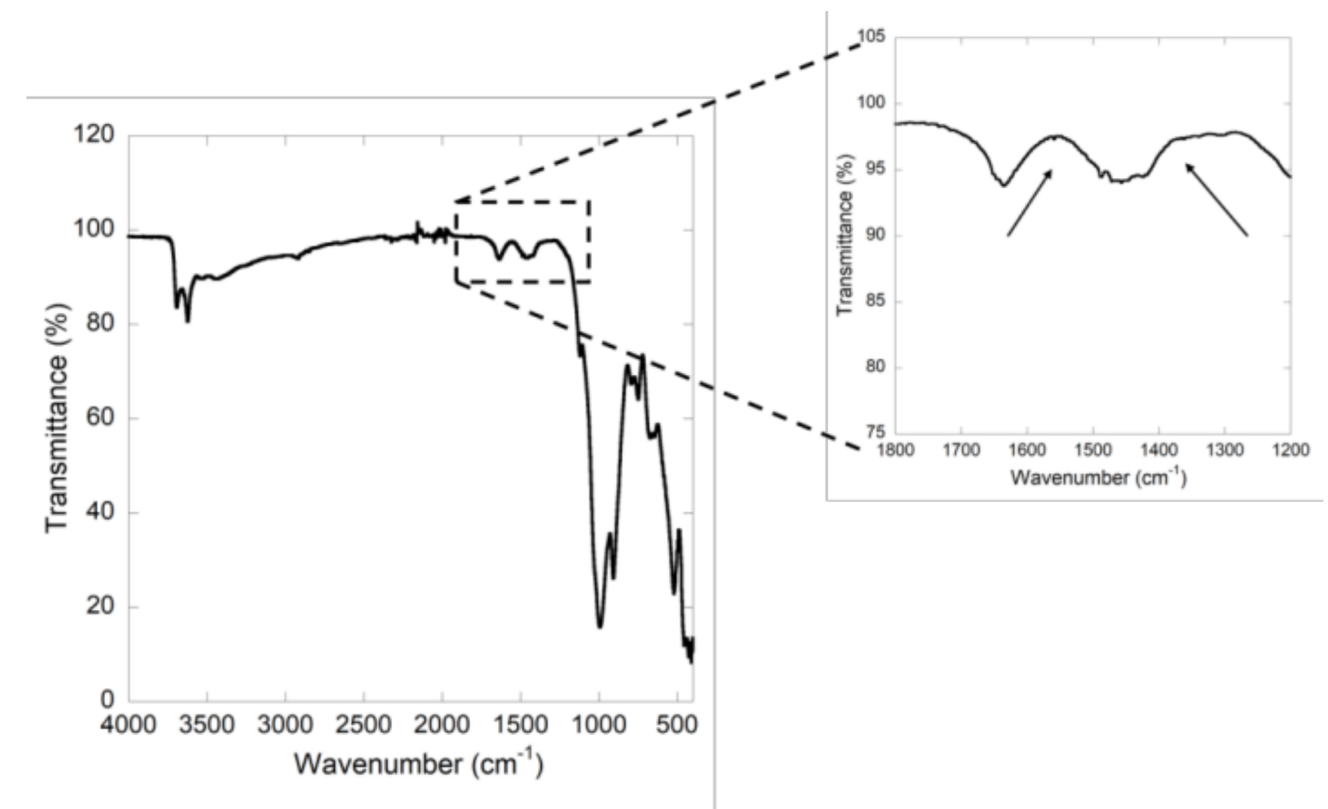

(a)

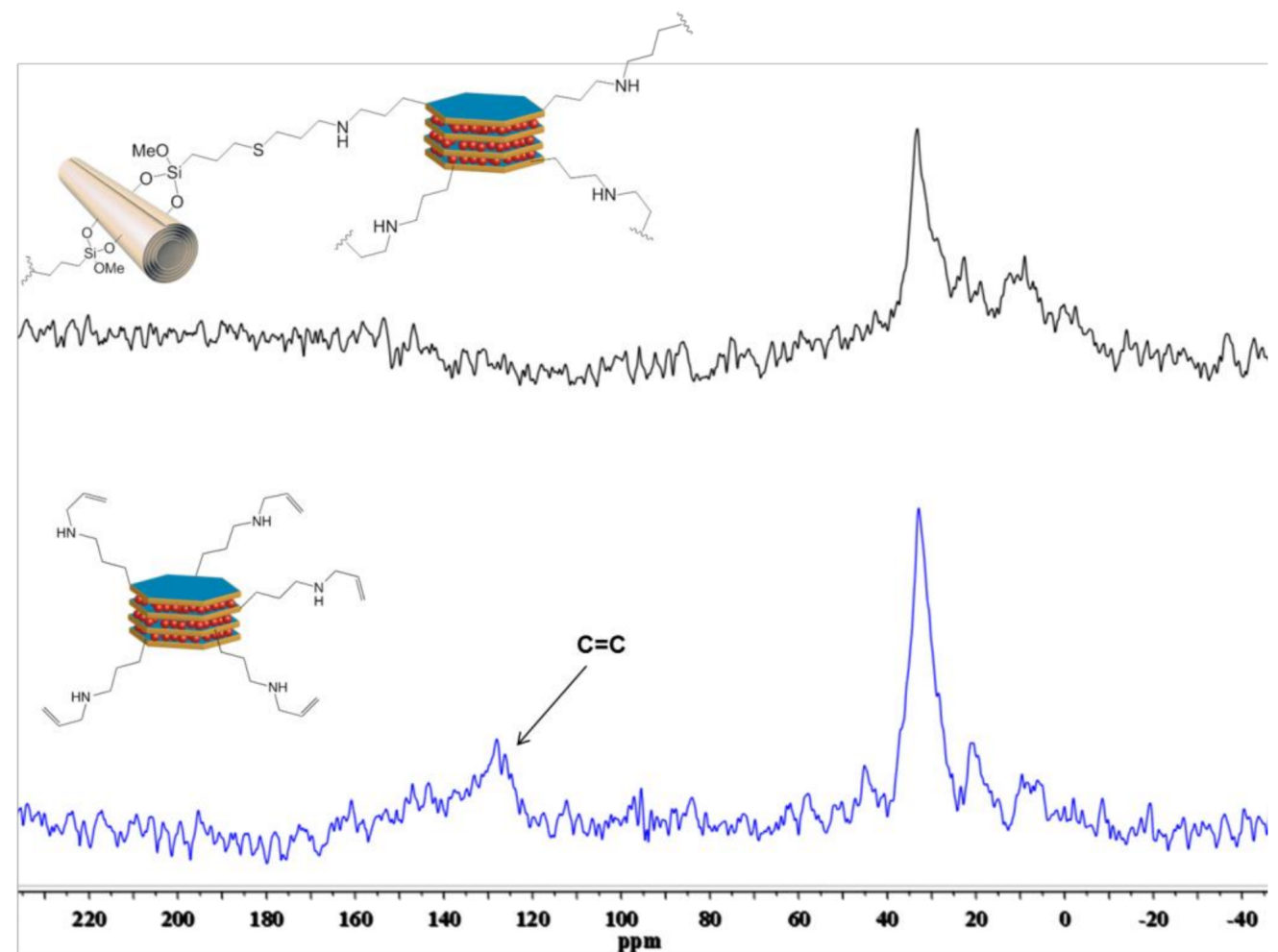

(b)

Figure 2. (a) FTIR spectrum of HNTs-Ht nanomaterial; (b) solid state ${ }^{13} \mathrm{C}$ NMR spectra of Ht-Allyl (blue line) and Hal-Ht (black line) nanomaterials. 
Table 2. Hydrodynamic diameter and the $\zeta$-potential data.

\begin{tabular}{ccc}
\hline Material & Hydrodynamic Diameter $\mathbf{( n m )}$ & $\zeta$-Potential $(\mathbf{m} \mathbf{)})$ \\
\hline Ht & $432 \pm 80$ & $-36.9 \pm 1.9$ \\
Ht-Allyl & $484 \pm 89$ & $-15.0 \pm 1.4$ \\
HNTs & $421 \pm 91$ & $-34.4 \pm 1.8$ \\
HNTs-SH & $427 \pm 95$ & $-27.1 \pm 1.1$ \\
HNTs-Ht & $453 \pm 96$ & $-17.3 \pm 1.4 ;-35.5 \pm 1.7$ \\
\hline
\end{tabular}

As concerns the $\zeta$-potential, pure Ht and HNTs exhibited similar results. The covalent functionalization generated a decrease of the negative charge of both nanoparticles, while the covalently linked HNTs-Ht nanomaterial evidenced the presence of two populations with different surface charge. The surface charge reduction upon functionalization agrees with the less effective dissociation of the modified surfaces compared to the pristine ones. The presence of two separate values for the hybrid material could indicate the presence of clusters with different Ht/HNTs ratios and, therefore, a surface charge dominated by one of the two clays.

The morphology of the HNTs-Ht nanomaterial was imaged by TEM measurements. According to TEM images, the HNTs-Ht nanomaterial shows the typical nanorod-shaped structure of HNTs and the typical morphology of the hectorite (Figure S2).

The hybrid material showed a rather compact structure with a dimension of ca. $135 \mathrm{~nm}$ (Figure 3a,b). Considering an halloysite diameter of ca. $100 \mathrm{~nm}$ and a Ht one of ca. $25 \mathrm{~nm}$ [6], the result is coherent with the two clays bonded. Close observations revealed the presence of halloysite nanotubes covered by hectorite clay (black arrow in the figure). Further confirmation was obtained by combining the high angle annular dark field scanning TEM (HAADF-STEM) and energy dispersive $X$-ray analysis. The former gives Z-contrast images allowing discrimination between the light and heavy atoms compounds, the latter allows chemical identifications of the atoms. By the combination of the two techniques a 2D-chemical map of the different elements may be extracted. In Figure $3 \mathrm{c}$ are reported the elemental X-ray aluminum and magnesium maps. In the hybrid HNTs-Ht material a magnesium layer (attributed to the hectorite) is covering the halloysite nanotubes (as highlighted by the presence of aluminum).

EDX analysis (Figure $3 \mathrm{~d}$ and Figure S3) further confirmed the synthesis of the covalently linked clays highlighting the presence of all characteristic atoms of HNTs and Ht and the presence of sulfur and nitrogen in the nanomaterial deriving from the organic functionalities introduced. For comparison, we also acquired the HAADF-STEM (Figure 2e,f) of a physical mixture constituted by both clay precursors mixed in the same proportion used for the synthesis of HNTs-Ht nanomaterial. In this case the morphology of the mixture was different. Indeed, it is possible to distinguish the individual components, observing both halloysite nanotubes and hectorite randomly dispersed.

Both the physical mixture and HNTs-Ht nanomaterial show typical reflections of both HNTs and Ht [22]. After the covalent linkage between the two clays, all reflection peaks remain unchanged indicating that no structural variation occurs. Only an exception related to the basal reflection $\mathrm{d}_{001}$ of the hectorite was found. This shifts to lower $2 \theta$ values (ca. $4.84^{\circ} 2 \theta$ ) in comparison with the pristine clay mineral which showed a $\mathrm{d}_{001}$ basal reflection at $7.25^{\circ} 2 \theta$. These values indicated an increase in the interlayer space of $\mathrm{Ht}$ in the HNTs-Ht nanomaterial with respect to the pristine one, from 12.18 $\AA$ to $18.24 \AA$ according to Bragg's law, for pristine $\mathrm{Ht}$ and HNTs-Ht, respectively. This increase is due to the clay exfoliation as a consequence of the edges modification and a significant insertion of the silane coupling agent into the $\mathrm{Ht}$ interlayer according to previous reported for similar systems [23].

To gather information about the structure of the HNTs-Ht nanomaterial, XRD measurements were performed on both pristine clays, HNTs-Ht and on its physical mixture (Figure 4). 


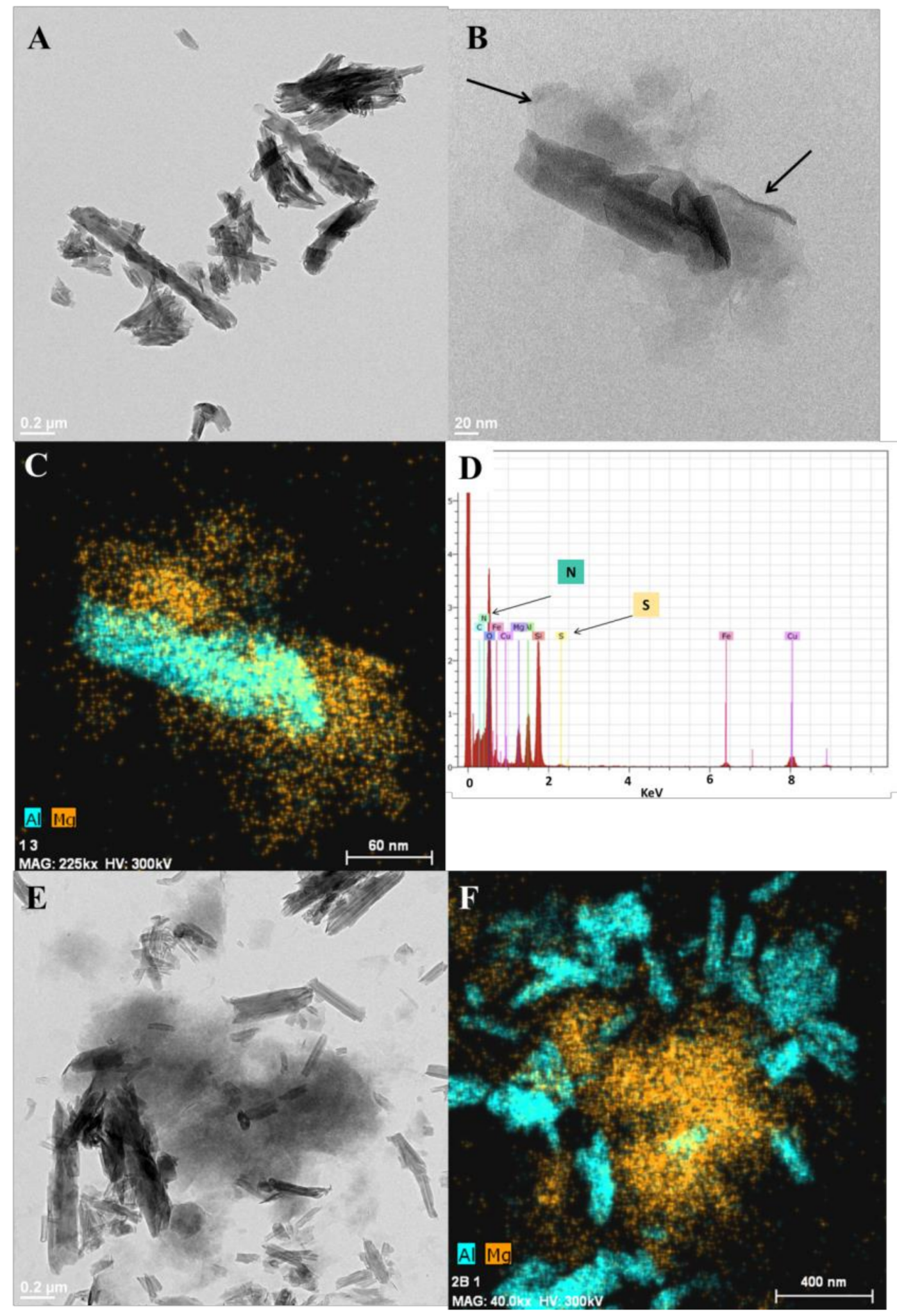

Figure 3. (a,b) TEM image of HNTs-Ht nanomaterial (scale bar 200 and $20 \mathrm{~nm}$, respectively); (c) corresponding EDX image of $\mathrm{Al}$ and $\mathrm{Mg}$ from STEM image (scale bar $60 \mathrm{~nm}$ ); (d) EDX spectrum of the HNTs-Ht nanomaterial; (e) TEM image of the physical mixture constituted by HNTs and Ht (scale bar $0.2 \mu \mathrm{m}$ ); and (f) the corresponding EDX image of $\mathrm{Al}$ and $\mathrm{Mg}$ from STEM image (scale bar $0.4 \mu \mathrm{m}$ ). 


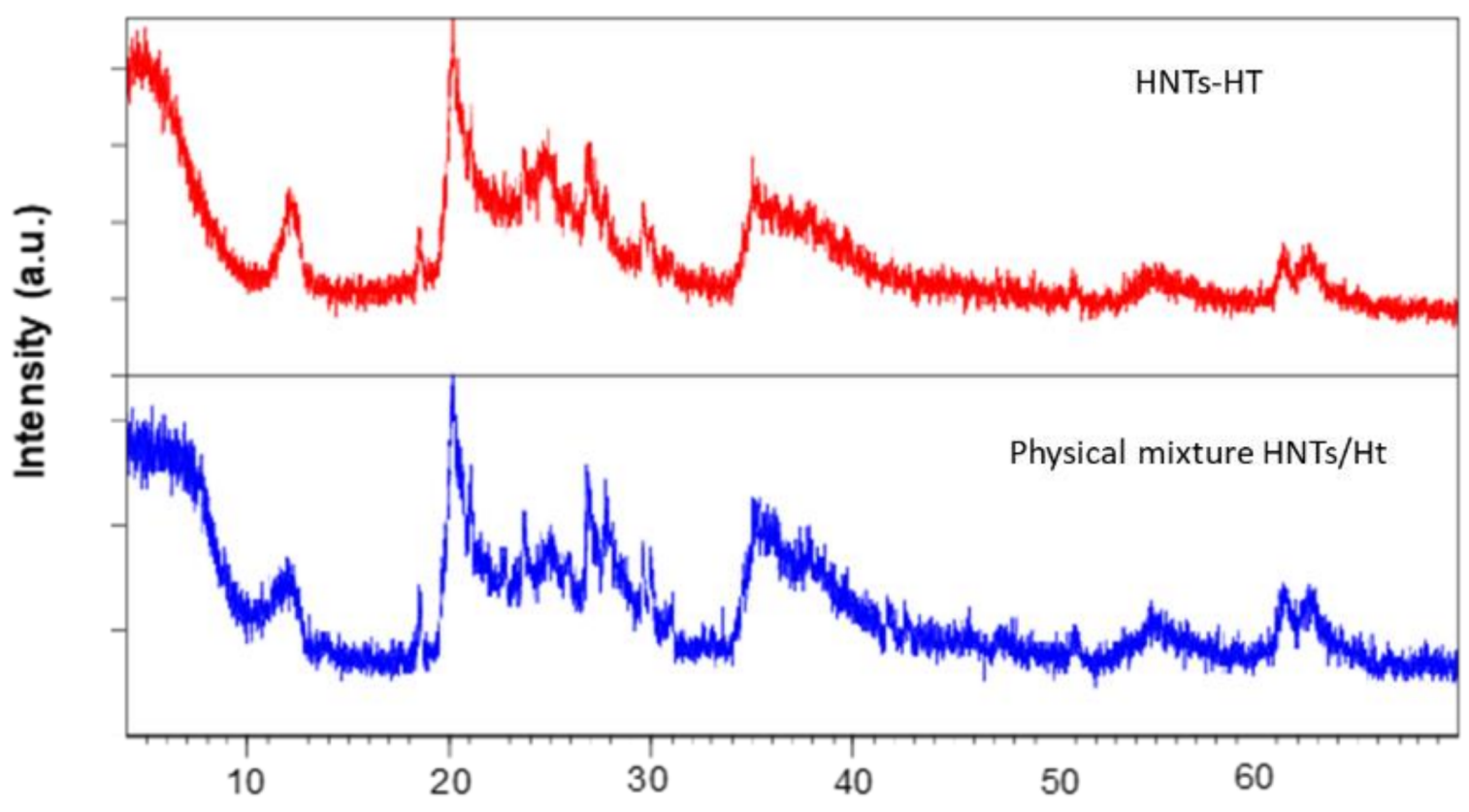

2 Theta

Figure 4. Comparison between the XRD patterns of HNTs/Ht physical mixture and HNTs-Ht nanomaterial.

\subsection{Adsorption Studies}

As a proof of concept, the adsorption ability of HNTs-Ht nanomaterial in comparison to the pristine clays was investigated by adsorption experiments towards ciprofloxacin and silver ions chosen as models (details of the ciprofloxacin properties are reported in SI) (Table 3).

Table 3. Adsorption ability of HNTs, Ht and HNTs-HT towards ciprofloxacin and $\mathrm{Ag}^{+}$ions.

\begin{tabular}{|c|c|c|c|c|}
\hline Entry & Sample & $\mathrm{mg}$ & $\begin{array}{c}\text { Ciprofloxacin } \\
(w t \%)^{a}\end{array}$ & $\begin{array}{c}\mathrm{Ag}^{+} \text {ions } \\
(\mathbf{w t} \%)^{\mathrm{b}}\end{array}$ \\
\hline 1 & HNTs & 16 & 2.6 & $<1$ \\
\hline 2 & $\mathrm{Ht}$ & 16 & $<1$ & 7 \\
\hline 3 & HNTs-Ht & 32 & 2.6 & 8 \\
\hline 4 & $\mathrm{HNTs} / \mathrm{Ht}$ & 32 & $<1$ & 7.6 \\
\hline
\end{tabular}

a Estimated by TGA, ${ }^{\text {b }}$ Estimated by $\mathrm{AgCl}$ test.

Experimental investigations performed by means of TGA and $\mathrm{AgCl}$ test (commonly adopted to estimate the amount of $\mathrm{Ag}^{+}$ions in solution by exploiting the low solubility of $\mathrm{AgCl}$ salt) highlighted that ciprofloxacin did not interact with pristine Ht (entry 2), on the contrary, it was encapsulated into the pristine HNTs lumen (entry 1), whereas silver ions preferentially interact with $\mathrm{Ht}$, presumable by cation exchange in the interlayer planes of the Ht particles as commonly reported for the clays belonging to the smectite group [24] (entry 2).

The HNTs covalently linked Ht showed the simultaneously adsorption of both ciprofloxacin and $\mathrm{Ag}^{+}$ions (entry 3). The characterization of the drugs loaded nanomaterial highlighted the presence of ca. $2.6 \mathrm{wt} \%$ of ciprofloxacin and ca. $8 \mathrm{wt} \%$ of $\mathrm{Ag}^{+}$ions, estimated by TGA (Figure S4) and $\mathrm{AgCl}$ test, respectively.

For comparison, the adsorption ability of the physical mixture of HNTs and Ht towards ciprofloxacin and $\mathrm{Ag}^{+}$ions, was also investigated (entry 4). Conversely to HNTs$\mathrm{Ht}$ nanomaterial, the physical mixture can efficiently adsorb $\mathrm{Ag}^{+}$ions, whereas no loading of ciprofloxacin occurs. The latter could be explained by the different disposition of the 
clays in the two cases. In particular, in the physical mixture the clays, presumably, arrange themselves to minimize repulsive interactions; Ht nanodisks could locate adjacent to the ends of HNTs by blocking the lumen as reported in literature both in the case of HNTs dispersions at different $\mathrm{pH}$ and mixture HNTs/Laponite ${ }^{\circledR}[25,26]$.

Therefore, it is possible to conclude that the HNTs-Ht nanomaterial, combining the properties of both clays, could be considered an efficient system for the co-delivery of species with different chemical characteristics.

\section{Conclusions}

In conclusion, we have reported a straightforward, simple and controllable method for the covalent linkage between two different clay minerals, namely halloysite nanotubes and hectorite. To reach this objective, hectorite was first functionalized in a two steps procedure. The physico-chemical characterization showed that pristine components retained their physico-chemical properties after the linkage. The morphology of the nanomaterial was imaged by TEM/HAADF technique. Finally, preliminary investigations were performed in order to verify the complementary properties of the nanomaterial in comparison to those of pristine clays towards the adsorption of ciprofloxacin and silver ions, chosen as models.

The designed nanomaterial demonstrated adsorption abilities similar to the pristine components separately, and consequently could be used as multi-functional or co-processed excipient, with improving manufacturing process of pharmaceutical products, including as for example, reduction of operating cost and improvement of final product quality. Furthermore, HNTs-Ht nanomaterial could be an alternative system for the simultaneous co-delivery of different antibacterial agents for the treatment of different patologies. It, indeed presents similar adsorption behavior of other systems commonly used, such as bentonite, fluorohectorite, montmorillonite, and hydroxyapatite, for the delivery of ciprofloxacin and silver ions [27-31].

Supplementary Materials: The following are available online at https:/ /www.mdpi.com/2079-499 1/11/2/506/s1, Figure S1. TGA curve of HNTs-Ht; Figure S2. TEM images of pristine HNTs and Ht; Figure S3. EDX spectrum of HNTs-Ht; physico-chemical properties of ciprofloxacin, Figure S4. TGA curve of HNTs, Ht and HNTs-Ht loaded with ciprofloxacin.

Author Contributions: Conceptualization: S.R., C.V.I.; methodology: S.R.; investigation: M.M., G.C., C.G.C., F.G.-V., G.L.; data curation: M.M., G.C., G.L.; writing—original draft preparation: M.M., S.R.; writing - review and editing: S.R.; supervision: C.V.I., S.R.; funding acquisition: C.V.I. All authors have read and agreed to the published version of the manuscript.

Funding: This research received no external funding.

Data Availability Statement: The data presented in this study are available on request from the corresponding author.

Acknowledgments: The work was financially supported by the University of Palermo. The work was carried out in the frame of the PON "AIM: Attrazione e Mobilità Internazionale" no. 1808223-1 project. Analyses performed in the University of Granada were financed by the project P18-RT-3786. Solid-state ${ }^{13} \mathrm{C}$ NMR spectra were provided by ATeN Center-University of Palermo.

Conflicts of Interest: The authors declare no conflict of interest.

\section{References}

1. Zhao, X.; Zhou, C.; Liu, M. Self-assembled structures of halloysite nanotubes: Towards the development of high-performance biomedical materials. J. Mater. Chem. B 2020, 8, 838-851.

2. Santos, A.C.; Pereira, I.; Reis, S.; Veiga, F.; Saleh, M.; Lvov, Y. Biomedical potential of clay nanotube formulations and their toxicity assessment. Expert Opin. Drug Deliv. 2019, 16, 1169-1182.

3. Massaro, M.; Colletti, C.G.; Guernelli, S.; Lazzara, G.; Liu, M.; Nicotra, G.; Noto, R.; Parisi, F.; Pibiri, I.; Spinella, C.; et al. Photoluminescent hybrid nanomaterials from modified halloysite nanotubes. J. Mater. Chem. C 2018, 6, 7377-7384.

4. Stavitskaya, A.; Batasheva, S.; Vinokurov, V.; Fakhrullina, G.; Sangarov, V.; Lvov, Y.; Fakhrullin, R. Antimicrobial applications of clay nanotube-based composites. Nanomaterials 2019, 9, 708. 
5. Stavitskaya, A.; Shakhbazova, C.; Cherednichenko, Y.; Nigamatzyanova, L.; Fakhrullina, G.; Khaertdinov, N.; Kuralbayeva, G.; Filimonova, A.; Vinokurov, V.; Fakhrullin, R. Antibacterial properties and in vivo studies of tannic acid-stabilized silver-halloysite nanomaterials. Clay Miner. 2020, 55, 112-119.

6. Massaro, M.; Noto, R.; Riela, S. Past, present and future perspectives on halloysite clay minerals. Molecules 2020, $25,4863$.

7. Riela, S.; Barattucci, A.; Barreca, D.; Campagna, S.; Cavallaro, G.; Lazzara, G.; Massaro, M.; Pizzolanti, G.; Salerno, T.M.G.; Bonaccorsi, P.; et al. Boosting the properties of a fluorescent dye by encapsulation into halloysite nanotubes. Dye. Pigment. 2021, 187, 109094.

8. Massaro, M.; Barone, G.; Barra, V.; Cancemi, P.; Di Leonardo, A.; Grossi, G.; Lo Celso, F.; Schenone, S.; Viseras Iborra, C.; Riela, S. Pyrazole[3,4-d]pyrimidine derivatives loaded into halloysite as potential cdk inhibitors. Int. J. Pharm. 2021, 120281. [CrossRef]

9. Jinhua, W.; Xiang, Z.; Bing, Z.; Yafei, Z.; Rui, Z.; Jindun, L.; Rongfeng, C. Rapid adsorption of cr (vi) on modified halloysite nanotubes. Desalination 2010, 259, 22-28.

10. Cataldo, S.; Lazzara, G.; Massaro, M.; Muratore, N.; Pettignano, A.; Riela, S. Functionalized halloysite nanotubes for enhanced removal of lead(ii) ions from aqueous solutions. Appl. Clay Sci. 2018, 156, 87-95.

11. Zhang, J.; Zhou, C.H.; Petit, S.; Zhang, H. Hectorite: Synthesis, modification, assembly and applications. Appl. Clay Sci. 2019, 177, 114-138.

12. Glotov, A.; Levshakov, N.; Stavitskaya, A.; Artemova, M.; Gushchin, P.; Ivanov, E.; Vinokurov, V.; Lvov, Y. Templated self-assembly of ordered mesoporous silica on clay nanotubes. Chem. Commun. 2019, 55, 5507-5510.

13. Li, S.; Mu, B.; Wang, X.; Kang, Y.; Wang, A. Fabrication of eco-friendly betanin hybrid materials based on palygorskite and halloysite. Materials 2020, 13, 4649.

14. Lisuzzo, L.; Wicklein, B.; Lo Dico, G.; Lazzara, G.; del Real, G.; Aranda, P.; Ruiz-Hitzky, E. Functional biohybrid materials based on halloysite, sepiolite and cellulose nanofibers for health applications. Dalton Trans. 2020, 49, 3830-3840.

15. Massaro, M.; Colletti, C.G.; Fiore, B.; La Parola, V.; Lazzara, G.; Guernelli, S.; Zaccheroni, N.; Riela, S. Gold nanoparticles stabilized by modified halloysite nanotubes for catalytic applications. Appl. Organomet. Chem. 2019, 33, e4665.

16. Colletti, C.G.; Massaro, M.; Lazzara, G.; Cavallaro, G.; Milioto, S.; Pibiri, I.; Noto, R.; Riela, S. Synthesis, characterization and study of covalently modified triazole laponite ${ }^{\circledR}$ edges. Appl. Clay Sci. 2020, 187, 105489.

17. de Castro Silva, F.; Brandão Lima, L.C.; Silva-Filho, E.C.; Fonseca, M.G.; Jaber, M. Through alizarin-hectorite pigments: Influence of organofunctionalization on fading. Colloids Surf. Physicochem. Eng. Asp. 2020, 587, 124323.

18. Lisuzzo, L.; Cavallaro, G.; Milioto, S.; Lazzara, G. Effects of halloysite content on the thermo-mechanical performances of composite bioplastics. Appl. Clay Sci. 2020, 185, 105416.

19. Massaro, M.; Cavallaro, G.; Colletti, C.G.; D'Azzo, G.; Guernelli, S.; Lazzara, G.; Pieraccini, S.; Riela, S. Halloysite nanotubes for efficient loading, stabilization and controlled release of insulin. J. Colloid Interface Sci. 2018, 524, 156-164.

20. Massaro, M.; Colletti, C.G.; Buscemi, G.; Cataldo, S.; Guernelli, S.; Lazzara, G.; Liotta, L.F.; Parisi, F.; Pettignano, A.; Riela, S. Palladium nanoparticles immobilized on halloysite nanotubes covered by a multilayer network for catalytic applications. New $\mathrm{J}$. Chem. 2018, 42, 13938-13947.

21. Cavallaro, G.; Lazzara, G.; Milioto, S. Exploiting the colloidal stability and solubilization ability of clay nanotubes/ionic surfactant hybrid nanomaterials. J. Phys. Chem. C 2012, 116, 21932-21938.

22. Pasbakhsh, P.; Churchman, G.J.; Keeling, J.L. Characterisation of properties of various halloysites relevant to their use as nanotubes and microfibre fillers. Appl. Clay Sci. 2013, 74, 47-57.

23. Vo, V.S.; Mahouche-Chergui, S.; Nguyen, V.H.; Naili, S.; Carbonnier, B. Crucial role of covalent surface functionalization of clay nanofillers on improvement of the mechanical properties of bioepoxy resin. ACS Sustain. Chem. Eng. 2019, 7, 15211-15220.

24. Horue, M.; Cacicedo, M.L.; Fernandez, M.A.; Rodenak-Kladniew, B.; Torres Sánchez, R.M.; Castro, G.R. Antimicrobial activities of bacterial cellulose-silver montmorillonite nanocomposites for wound healing. Mater. Sci. Eng. C 2020, 116, 111152.

25. Joo, Y.; Sim, J.H.; Jeon, Y.; Lee, S.U.; Sohn, D. Opening and blocking the inner-pores of halloysite. Chem. Commun. 2013, 49, $4519-4521$.

26. Massaro, M.; Buscemi, G.; Arista, L.; Biddeci, G.; Cavallaro, G.; D'Anna, F.; Di Blasi, F.; Ferrante, A.; Lazzara, G.; Rizzo, C.; et al. Multifunctional carrier based on halloysite/laponite hybrid hydrogel for kartogenin delivery. ACS Med. Chem. Lett. 2019, 10, 419-424.

27. Peighambardoust, S.J.; Zahed-Karkaj, S.; Peighambardoust, S.H.; Ebrahimi, Y.; Peressini, D. Characterization of carboxymethyl cellulose-based active films incorporating non-modified and ag or cu-modified cloisite $30 \mathrm{~b}$ and montmorillonite nanoclays. Iran Polym. J. 2020, 29, 1087-1097.

28. Gulen, B.; Demircivi, P. Synthesis and characterization of montmorillonite/ciprofloxacin/tio2 porous structure for controlled drug release of ciprofloxacin tablet with oral administration. Appl. Clay Sci. 2020, 197, 105768.

29. Maged, A.; Kharbish, S.; Ismael, I.S.; Bhatnagar, A. Characterization of activated bentonite clay mineral and the mechanisms underlying its sorption for ciprofloxacin from aqueous solution. Environ. Sci. Pollut. Res. 2020, 27, 32980-32997.

30. Valdés, L.; Pérez, I.; de Ménorval, L.C.; Altshuler, E.; Fossum, J.O.; Rivera, A. A simple way for targeted delivery of an antibiotic: In vitro evaluation of a nanoclay-based composite. PLOS ONE 2017, 12, e0187879.

31. Xiong, Z.-C.; Yang, Z.-Y.; Zhu, Y.-J.; Chen, F.-F.; Zhang, Y.-G.; Yang, R.-L. Ultralong hydroxyapatite nanowires-based paper co-loaded with silver nanoparticles and antibiotic for long-term antibacterial benefit. ACS Appl. Mater. Interf. 2017, 9, 22212-22222. 